\title{
A retro-odontoid pseudotumor treated with fixation and tumor resection by the lateral approach: illustrative case
}

\author{
Yoshiaki Oda, MD, PhD, ${ }^{1}$ Takamitsu Tokioka, MD, PhD, ${ }^{2}$ and Toshifumi Ozaki, MD, PhD ${ }^{1}$ \\ ${ }^{1}$ Department of Orthopedic Surgery, Okayama University Hospital, Okayama, Japan; and ${ }^{2}$ Department of Orthopedic Surgery, Kochi Health Sciences Center, Kochi, Japan
}

BACKGROUND A retro-odontoid pseudotumor is not a condition that requires resection. However, pathological diagnosis is required when a tumor such as a meningeal tumor or chordoma is suspected. The authors report a case of a large lesion treated with posterior fixation and tumor resection using a lateral approach.

OBSERVATIONS A 77-year-old man visited the authors' department complaining of neck pain and decreased dexterity of the upper extremities. Magnetic resonance imaging showed a large, beak-shaped lesion behind the dens and severe compression of the spinal cord. Surgery consisted of occipitocervical-C2 fixation, followed by tumor resection with a left lateral approach. The pathological diagnosis was consistent with a retro-odontoid pseudotumor. The tumor was resected to a relatively large extent and shrank over time, leading to complete disappearance.

LESSONS Pathological examination is also possible with a posterior approach if the tumor can be reached through the lateral edge of the dura. In that situation, the amount of resection is limited, and there is a risk of spinal cord compression. Intradural dissemination of tumors is a concern with the transdural approach. If tumor resection by the posterior approach is difficult, the lateral approach can facilitate tumor resection.

https://thejns.org/doi/abs/10.3171/CASE20160

KEYWORDS retro-odontoid pseudotumor; lateral approach; tumor resection

The resection of neoplastic pathology at the craniocervical spine junction is technically difficult. A retro-odontoid pseudotumor is a mass that compresses the spinal cord at the craniocervical spine junction; resection of the compressed lesion is difficult. Retro-odontoid pseudotumors can be caused by inflammatory diseases such as rheumatoid arthritis or chronic instability between the C1 and C2 vertebrae. In cases resulting from atlantoaxial instability, good results have been reported with fusion alone, and tumor resection is not always necessary. ${ }^{1}$ Pathological examination should be performed for differentiated diseases, including neoplastic lesions such as meningioma and chordoma. We report a case in which a lateral approach to the upper cervical spine in addition to posterior fusion was used because of the large size of the retro-odontoid pseudotumor. Magnetic resonance imaging (MRI) performed 5 months after the operation showed that the pseudotumor had completely disappeared.

\section{Illustrative Case}

History and Examination

A 77-year-old man experienced neck pain and headache for 1 month and consulted his family doctor. MRI of the brain was per- formed, revealing a compression lesion at the craniocervical spine junction. Therefore, the patient was referred to our department. During medical examination, the patient was able to use chopsticks, but his fine motor movements were impaired, making it difficult to remove coins from a wallet. His gait was mildly spastic but self-sustaining. A radiograph showed degeneration of the subaxial spine, and a dynamic radiograph showed that subaxial spine mobility was reduced and $\mathrm{C} 1-2$ mobility was increased (Fig. $1 \mathrm{~A}-\mathrm{C}$ ). The patient's atlantodental interval was $3.3 \mathrm{~mm}$. MRI showed a large, beak-shaped lesion behind the dens and severe compression of the spinal cord (Fig. 1E and F). Surgical treatment was indicated, but 3 days after the patient visited our department, he burned his face and limbs, and treatment was interrupted for about 1.5 months. When he revisited the outpatient center, his symptoms had progressed: His gait was unstable, and he required handrails to walk upstairs. He complained of a headache in the sitting position and frequent urination. Computed tomography (CT) showed bone components in the compression lesion behind the dens (Fig. 1F and $G$ ). The degree of compression was severe, and we decided to perform tumor resection and subsequent pathological examination. 

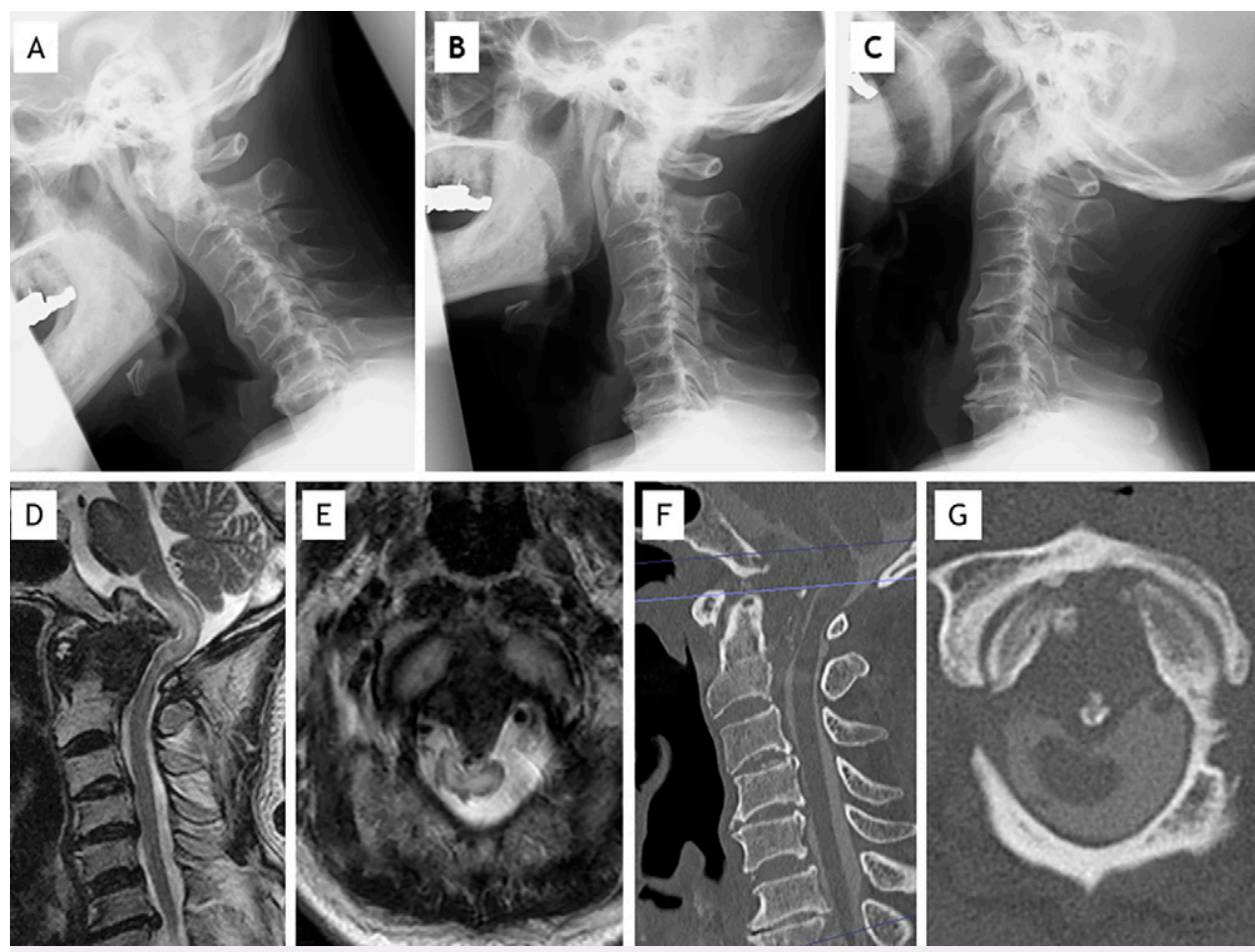

FIG. 1. Preoperative imaging studies. Radiographs in the flexion position (A), neutral position (B), and extension position (C) show reduced mobility of the subaxial spine. The atlantodental interval was $3.3 \mathrm{~mm}$. MRI showed a large, beak-shaped lesion behind the dens and severe compression of the spinal cord ( $D$ and $\mathbf{E})$. CT showed bone components in the compression lesion behind the dens ( $F$ and $\mathbf{G})$.

\section{Operation, Pathological Findings, and Postoperative Course}

The operation was performed with the patient in the prone position under general anesthesia and the head held with a Mayfield clamp.
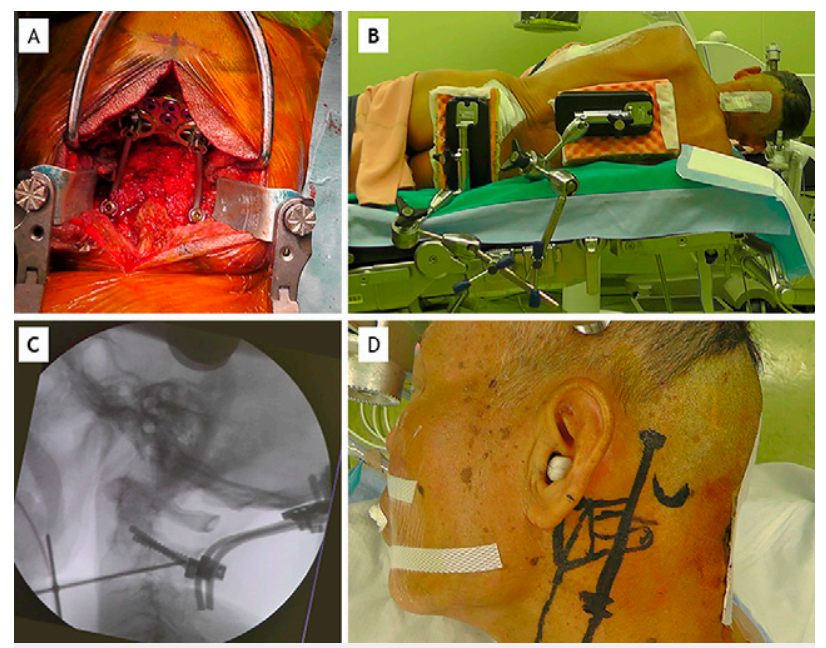

FIG. 2. Intraoperative findings. O-C2 fusion was performed (A). The patient's position was changed from the prone position to the lower right lateral decubitus position (B). The locations of $\mathrm{C} 1$ and $\mathrm{C} 2$ were marked on the skin with reference to the fluoroscopy (C), and the 10-cm skin incision was made to pass over the sternocleidomastoid muscle (D).
The alignment of the craniocervical spine was confirmed by fluoroscopy, and the position was fixed. A skin incision was made from the occipital tubercle to the C2 spinous process, and surgery was started. A C2 pars screw was inserted under the navigation guide, and the occipital plate was placed and then connected with a titanium rod. After decortication of the C1 posterior arch, the C2 lamina, and the occipital bone, cancellous bone harvested from the ilium was transplanted and occipitocervical (0)-C2 fusion was performed (Fig. 2A).

The patient's position was changed from the prone position to the lower right lateral decubitus position (Fig. 2B). The location of $\mathrm{C} 1$ and $\mathrm{C} 2$ was marked on the skin under fluoroscopy (Fig. 2C). A 10-cm skin incision was made to pass over the sternocleidomastoid muscle (Fig. 2D).

The sternocleidomastoid muscle was detached from the occipital bone and retracted anteriorly, and the splenius capitis muscle was moved posteriorly (Fig. 3A). The $\mathrm{C} 1$ transverse process and the large occipital triangle were identified by palpation. The inferior oblique muscle attached to the $\mathrm{C} 1$ transverse process was removed, and it was exposed using an electric cautery device (Fig. 3B). The transverse foramen of $\mathrm{C} 1$ was opened with a high-speed drill to protect the vertebral arteries (Fig. 3C). After the boundary between the anterior part of the dura mater and the compressive lesion became visible, the lesion was resected with forceps and submitted for rapid pathological examination (Fig. 3D). Intraoperative rapid pathology did not reveal a neoplastic lesion, so as much of the lesion was resected as possible. After irrigation and confirming hemostasis, a negative pressure drain was installed, and the muscle layer was sutured to close the wound (Fig. 4A and B). Pathological examination revealed fibrous tissue with a mucous-like substrate. Partially necrotic changes and fibrous precipitation 

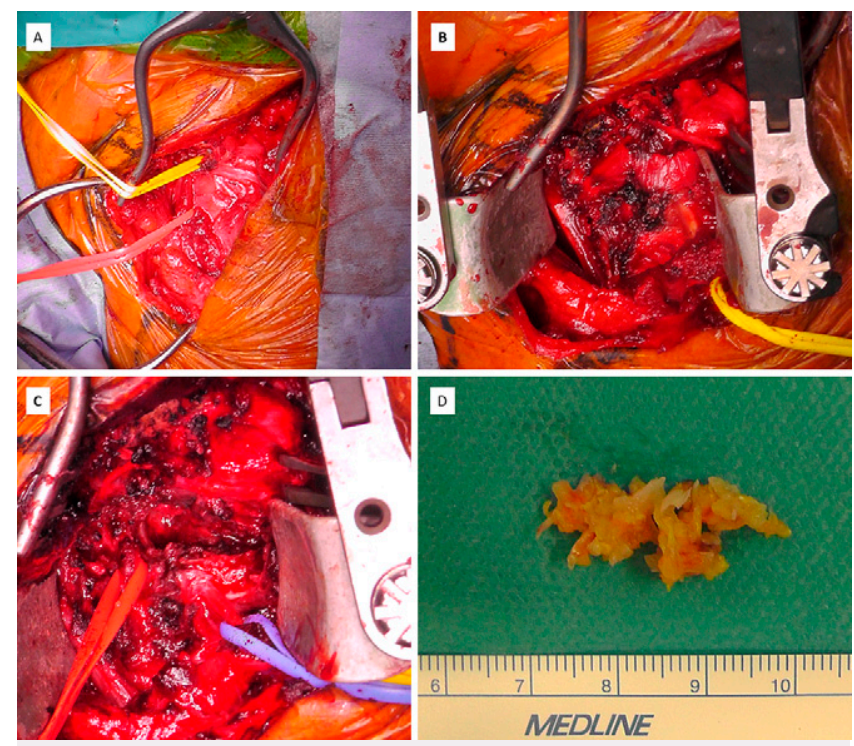

FIG. 3. The sternocleidomastoid muscle was detached from the occipital bone and retracted anteriorly, and the splenius capitis muscle was moved posteriorly (A). The inferior oblique muscle was detached to expose the C1 transverse process (B). The transverse foramen of $\mathrm{C} 1$ was opened with a high-speed drill (C) to protect the vertebral arteries (red tape). The resected lesion was submitted for pathological examination (D). were also observed. Congo red staining was weakly positive and showed amyloid in the tissue.

After the operation, there was no neurological deterioration, no paralysis of the accessory nerve or facial nerve related to the approach, and no sensory impairment behind the auricle. It was recommended that the patient use a collar when leaving the bed. CT performed 1 week after the operation revealed that the amount of excised $\mathrm{C} 1$ was the posterior half of the $\mathrm{C} 1$ transverse foramen (Fig. $4 \mathrm{C}$ and D). The patient did not experience postoperative complications and was able to walk independently at the time of discharge. MRI performed 5 months after the operation showed that the retro-odontoid pseudotumor had completely disappeared (Fig. 4E and F). The patient provided informed consent to participate in this research and to allow the publication of this case report and the accompanying images.

\section{Discussion}

It has been reported that a retro-odontoid pseudotumor is associated with inflammatory diseases such as rheumatoid arthritis or with chronic instability at C1-2. Differential diagnosis includes tumors such as meningioma, chordoma, and osteochondroma. ${ }^{2}$ In this case, the lesion was large; therefore, we thought pathological examination should be performed to make a diagnosis.

Surgical procedures for retro-odontoid pseudotumor include $\mathrm{C} 1$ posterior arch resection, ${ }^{3}$ fusion, or a combination of the two. ${ }^{1}$ Fusion surgery includes $\mathrm{C} 1-2$ fixation and $\mathrm{O}-\mathrm{C} 2$ fixation. The transoral, transdural, and lateral approaches have all been reported as surgical techniques for resection of retro-odontoid pseudotumors. ${ }^{4-6}$
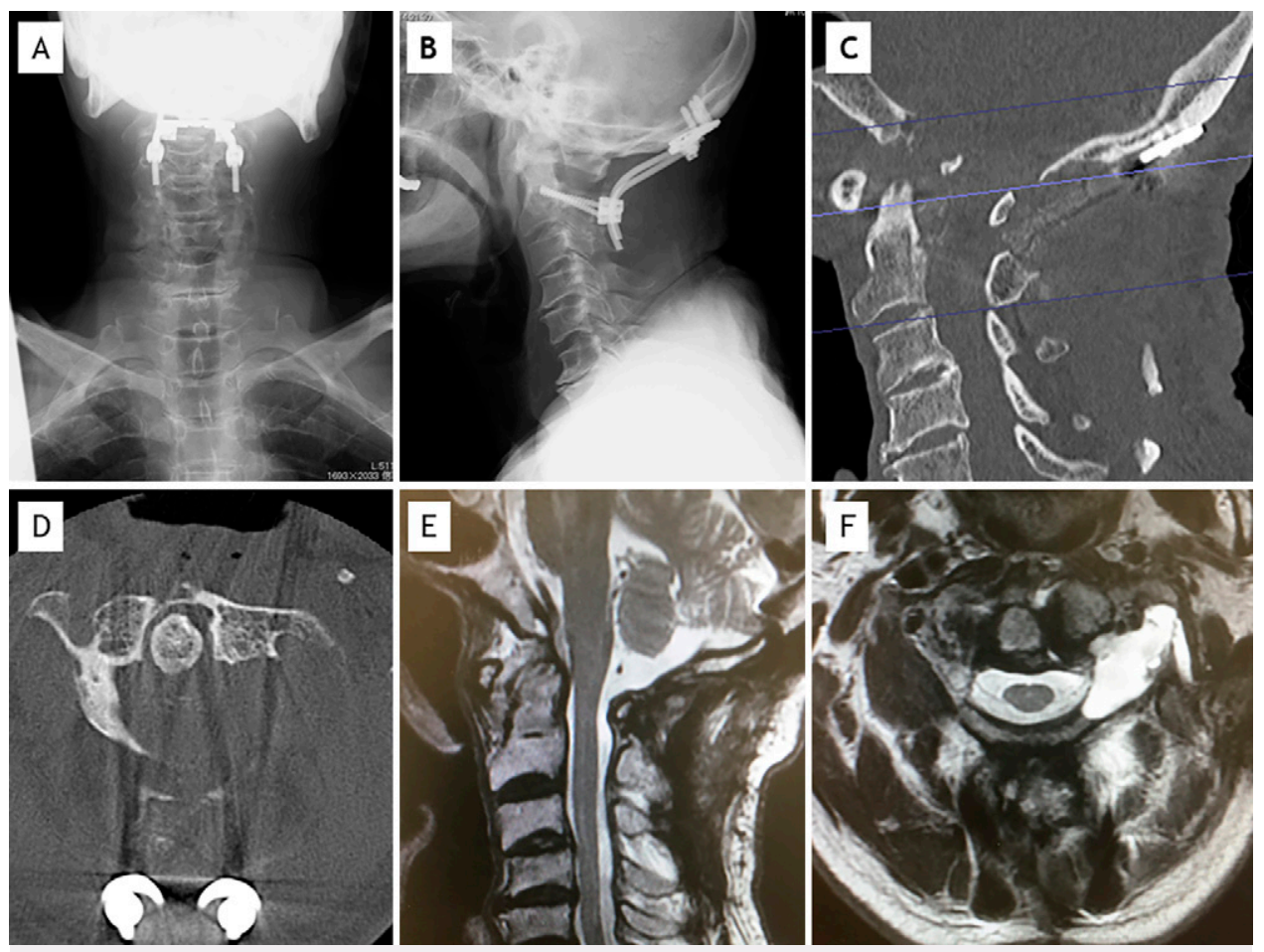

FIG. 4. Postoperative imaging studies. Anteroposterior (A) and lateral (B) radiographs. CT performed 1 week after the operation revealed that the amount of excised $\mathrm{C} 1$ was the posterior half of the $\mathrm{C} 1$ transverse foramen (C and D). MRI performed 5 months after the operation showed that the retro-odontoid pseudotumor had completely disappeared ( $\mathrm{E}$ and $\mathbf{F})$. 
The lateral cervical approach, unlike the posterior approach, is less familiar to spine surgeons. The lateral approach is an exacting procedure because of the narrow and deep surgical field and the risk of some nerve injury. However, the lateral approach allows the surgeon to safely approach the craniovertebral junction by moving and protecting the vertebral artery from the transverse foramen of $\mathrm{C} 1$. There are no reports of tumor resection from a lateral approach combined with fusion surgery.

\section{Observations}

We selected fusion surgery because fixation is effective for chronic instability in the case of retro-odontoid pseudotumor, and there is concern about instability after aggressive resection of the tumor in the case of nonpseudotumor. We decided to perform tumor resection using the lateral approach because both resection of enough volume of tumor for pathological examination and direct decompression of the spinal cord were possible. Surgery consisted of O-C2 fixation, followed by tumor resection with a left lateral approach. The tumor was resected in a relatively large amount. The tumor shrank over time, leading to complete disappearance.

\section{Lessons}

The merits of the lateral approach are as follows: (1) A larger amount of tumor can be resected; (2) the compression lesion in the anterior spinal cord can be resected directly; (3) the tumor is resected without requiring the compressive maneuver for the spinal cord; (4) the C1 posterior arch can be preserved, which is advantageous for bone fusion; and (5) there is no cerebrospinal fluid leakage because the dura mater is not opened. If tumor resection by the posterior approach is difficult, the lateral approach facilitates tumor resection and decreases spinal cord damage.

\section{References}

1. Barbagallo GMV, Certo F, Visocchi M, et al. Disappearance of degenerative, non-inflammatory, retro-odontoid pseudotumor following posterior C1-C2 fixation: case series and review of the literature. Eur Spine J. 2013;22(suppl 6):S879-S888.

2. Crockard HA, Sett $P$, Geddes JF, et al. Damaged ligaments at the craniocervical junction presenting as an extradural tumour: a differential diagnosis in the elderly. J Neurol Neurosurg Psychiatry. 1991;54(9):817-821.

3. Suetsuna $F$, Narita $H$, Ono A, Ohishi $H$. Regression of retroodontoid pseudotumors following C-1 laminoplasty. Report of three cases. J Neurosurg Spine. 2006;5(5):455-460.

4. Moskovich R, Crockard HA. Posttraumatic atlanto-axial subluxation and myelopathy. Efficacy of anterior decompression. Spine (Phila Pa 1976). 1990;15(6):442-447.

5. Fujiwara $Y$, Manabe H, Sumida T, et al. Microscopic posterior transdural resection of cervical retro-odontoid pseudotumors. J Spinal Disord Tech. 2015;28(10):363-369.

6. Oohori Y, Seichi A, Kawaguchi H, et al. Retroodontoid pseudotumor resected by a high cervical lateral approach in a rheumatoid arthritis patient: a case report. J Orthop Sci. 2004;9(1):90-93.

\section{Disclosures}

The authors report no conflict of interest concerning the materials or methods used in this study or the findings specified in this paper.

\section{Author Contributions}

Conception and design: Oda, Tokioka. Acquisition of data: Oda, Tokioka. Analysis and interpretation of data: Tokioka. Drafting the article: Oda. Approved the final version of the manuscript on behalf of all authors: Oda. Administrative/technical/material support: Tokioka. Study supervision: Tokioka, Ozaki.

\section{Correspondence}

Yoshiaki Oda: Okayama University Hospital, Okayama, Japan. odaaaaaaamn@yahoo.co.jp. 